\title{
Periocular Region
}

National Cancer Institute

\section{Source}

National Cancer Institute. Periocular Region. NCI Thesaurus. Code C62645.

The area surrounding the eye. 